The Astrophysical Journal, Vol. 156, April 1969

(C) 1969. The University of Chicago. All rights reserved. Printed in U.S.A.

\title{
SPECTROPHOTOMETRY AND INFRARED PHOTOMETRY OF BL LACERTAE
}

\author{
J. B. Oke and G. Neugebauer \\ Mount Wilson and Palomar Observatories, Carnegie Institution of Washington, \\ California Institute of Technology \\ AND \\ E. E. BECKLIN \\ California Institute of Technology \\ Received February 17, 1969
}

\section{ABSTRACT}

The absolute spectral-energy distribution of BL Lacertae from 0.315 to $2.2 \mu$ indicates that the optical radiation might be a continuation of the observed radio synchrotron flux. The very steep slope of the spectral-energy distribution suggests that the object is not a quasi-stellar radio source. Only one very faint spectral feature may exist, and no redshift can be derived.

\section{INTRODUCTION}

Recently the variable star BL Lacertae has been suggested as the optical identification for the radio source VRO 42.22.01 (Schmitt 1968; MacLeod and Andrew 1968). The star is highly variable, with a range in the blue from 13.0 to 16.1 mag (Schewick 1941; Semakin 1955). Slit spectra of the star show only a continuous spectrum with no emission or absorption features (van den Bergh 1969). Since the available data on this star have suggested that the radiation could be synchrotron radiation and that the object might be a quasi-stellar radio source, spectrophotometric and infrared observations have been obtained.

\section{OBSERVATIONS}

Photoelectric spectrophotometric observations were made on December 13, 1968, with 18 channels of the new multichannel spectrometer attached at the Cassegrain focus of the 200-inch Hale telescope (Oke 1969). These observations were made from 3150 to $5550 \AA$ with a band pass of $20 \AA$ and from 5660 to $8140 \AA$ with a band pass of $40 \AA$. Observations were reduced to absolute fluxes based on the absolute calibration of $a$ Lyrae given by Oke (1964), except that all points at wavelengths less than the Balmer limit were made fainter by 6 per cent. It was evident from the reductions that the object changed in brightness during the course of the 44 minutes of observations. Since measurements overlapped in wavelength, it was possible to estimate the magnitude changes. Corrections varying smoothly from 0.00 to $0.10 \mathrm{mag}$ were applied, with the assumption that the color remained always the same. All the observations are plotted in Figure 1. The ordinate is $\log f_{\nu}$, where $f_{\nu}$ is the flux at frequency $\nu\left(\mathrm{W} \mathrm{m}^{-2} \mathrm{~Hz}^{-1}\right)$; the abscissa is $\log \nu$. Standard deviations from $\log \nu=14.56$ to $\log \nu=14.70$ are 0.008 , and from $\log \nu=14.70$ to $\log \nu=14.93$ they are 0.020 ; above $\log \nu=14.70$, the errors gradually increase to 0.04 . The average visual magnitude was 15.1. Infrared observations at $1.65 \mu$ $(1.5-1.8 \mu)$ and $2.2 \mu(2.0-2.4 \mu)$ were made on August 7, 1968, at the 200-inch telescope, with the use of techniques described elsewhere (Neugebauer et al. 1969). These data are also shown in Figure 1, along with standard-deviation error bars. 


\section{DISCUSSION}

The spectrophotometric observations are ideal for finding broad, weak emission or absorption features. The only evidence for any such feature is a possible weak emission line at $\log \nu=14.79(4860 \AA)$. The apparent coincidence with $\mathrm{H} \beta$ cannot be meaningful, since there is no evidence for $\mathrm{H} a$ or $\mathrm{H} \gamma$. Identification with redshifted lines also appears very unlikely, since any reasonable identification would imply that other lines should have been observed. An apparent weak absorption feature near $\log \nu=14.86$ is probably not real. In the blue the energy distribution agrees with the three fluxes computed by Visvanathan (1969) from his $U B V$ measurements; in the red, from $\log \nu=14.55$ to $\log$ $\nu=14.70$, the fluxes also agree but are larger by 0.08 in $\log f_{\nu}$.

The observed energy distribution must be corrected for reddening. Since BL Lac lies in the direction of the I Lacertae association, the same visual absorption of 0.31 mag as determined by Crawford (1961) for this association has been adopted. The corrected

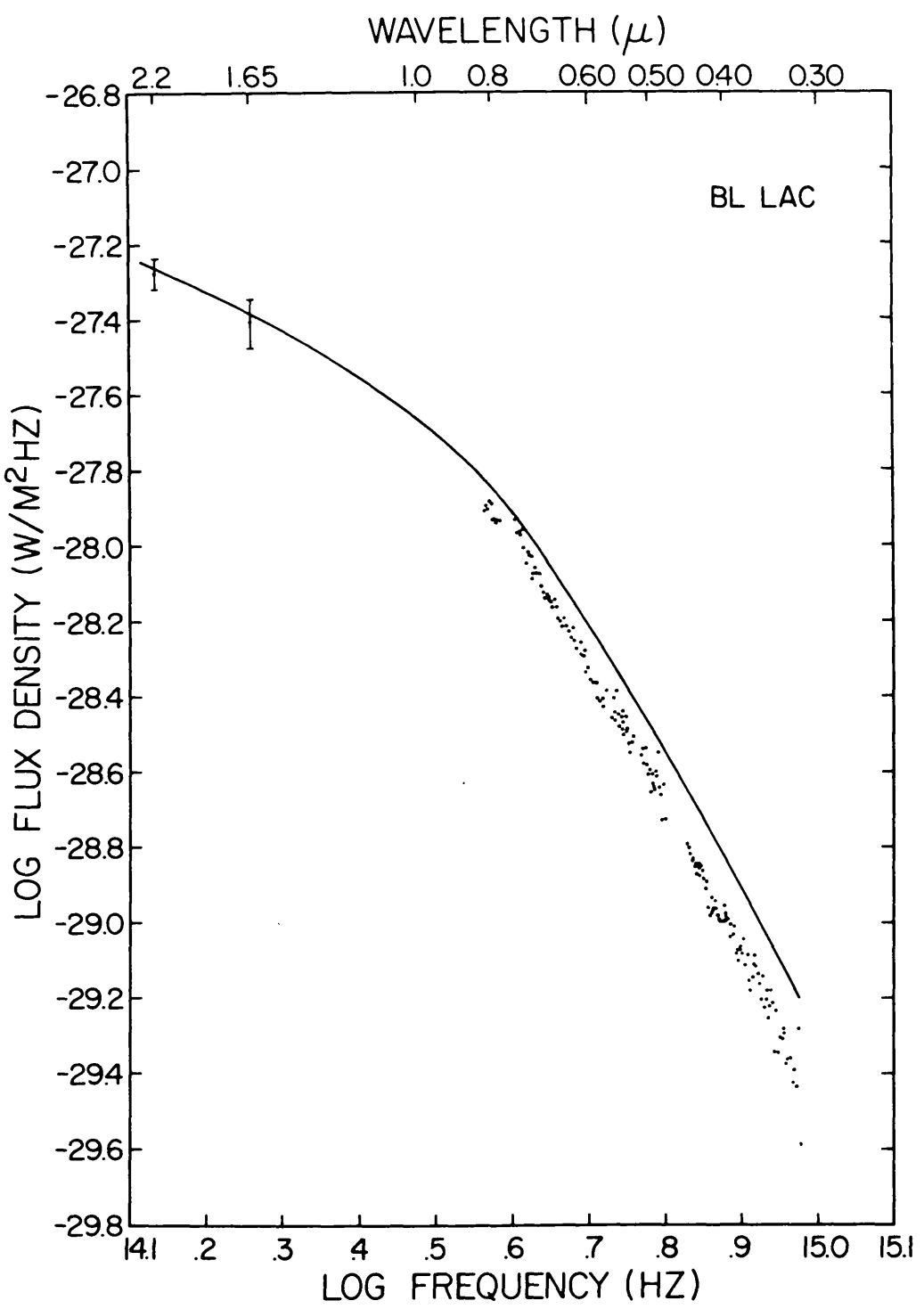

Fig. 1.-Spectral-energy distribution of BL Lac from 0.31 to $2.2 \mu$. Solid line represents energy distribution after a correction for $0.31 \mathrm{mag}$ of visual absorption has been applied. 
energy distribution, using the reddening law of Whitford (1958), is shown by the solid curve in Figure 1. For $\log \nu>14.55$, the slope $d\left(\log f_{v}\right) / d(\log \nu)$ is -3.6 and is nearly constant. In the infrared the slope is definitely less. This slope is twice that for the reddest known quasi-stellar sources (Oke, Neugebauer, and Becklin 1969), suggesting that this may be a different type of object.

The data presented in Figure 1 were, as noted, obtained on dates separated by about four months, and thus they may not, in fact, be representative of the continuum at any one time. The variability of BL Lac, however, occurs in bursts (Véron and Bertaud 1968), and thus it is probable that both observations were made in the quiescent state of BL Lac. Moreover, the slope derived from the infrared points is clearly different from that observed in the visible, although it is grossly consistent with an extension from the visual. The possibility that BL Lac changed in both magnitude and slope between the dates when the visual and infrared observations were made is unlikely but cannot be ruled out.

As discussed by Visvanathan (1969), the energy distribution can be interpreted as the cutoff spectral region of the synchrotron source which produces the radio observations. His polarization results tend to confirm this. The present measurements do not significantly modify his Figure 2, which shows both the radio and the optical data; the data of this Letter do, however, show the optical spectrum beginning to flatten near $\log \nu=14.3$. The large wavelength range now observed does rule out the possibility that this object is a thermal source at one blackbody temperature. If a blackbody or model atmosphere is fitted to the observed fluxes in the visual and blue, then the calculated fluxes are already dropping rapidly at 1.65 and $2.2 \mu$, contrary to observations.

\section{CONCLUSIONS}

Both the absolute spectral-energy distribution and the constancy of the magnitude and direction of polarization (Visvanathan 1969) suggest that BL Lac is a synchrotron source. The absence of identifiable spectral features leaves open the question of whether it is a quasi-stellar source. The very steep slope, however, suggests that it is not a quasistellar radio source.

We thank Dr. Wallace Sargent for calling our attention to BL Lac, and Mr. Gary Tuton for assistance in making the 200-inch observations.

This research was supported by grant NGL 05-002-007 from the National Aeronautics and Space Administration. The multichannel spectrometer and auxiliary equipment were provided by grant DA-ARO-D-31-124-G705 of the Advanced Research Projects Agency.

\section{REFERENCES}

Bergh, S. van den. 1969 (private communication).

Crawford, D. L. $1961, A p . J ., 133,860$.

MacLeod, J. M., and Andrew, B. H. 1968, A p. Letters, 1, 243.

Neugebauer, G., Oke, B., Becklin, E., and Garmire, G. 1969, A p. J., 155, 1.

Oke, J. B. 1964, $A$ p. J., 140, 689.

. 1969, Pub. A.S.P., 81, 11.

Oke, J. B., Neugebauer, G., and Becklin, E. E. 1969 (in preparation).

Schewick, H. V. 1941, Kl. Veröff. U. Sternw. Berlin-Babelsberg, No. 24, p. 103.

Schmitt, J. L. 1968, Nature, 218, 663.

Semakin, N. K. 1955, Variable Stars (Moscow), 10, 283.

Véron, P., and Bertaud, C. 1968, paper presented at the Fourth Texas Symposium on Relativistic Astrophysics.

Visvanathan, N. 1969, Ap. J. (Letters), 155, L133.

Whitford, A. E. 1958, A.J., 63, 201. 